\title{
An evaluation method of access capacity in distribution network Mingjun $\mathrm{Qi}^{1}$, Fengchuan $\mathrm{Nie}^{2, \mathrm{a}}$, Hua Leng ${ }^{1}$, Liang Zhu ${ }^{1}$, Minfang Peng ${ }^{2, \mathrm{~b}}$ \\ ${ }^{1}$ State Grid Hunan Electric Power Company, Changsha 410007, China \\ ${ }^{2}$ College of Electrical and Information Engineering, Hunan University, Changsha, 410082, China \\ aemail: fengchuanchina@163.com, bemail: pengminfang@hnu.edu.cn
}

\begin{abstract}
Keywords: marketing-distribution-dispatching information fusion; access capacity; the maximum
\end{abstract} installed access capacity

\begin{abstract}
In order to publish access capacity, warnings and loads in distribution network regularly, the article presents a method to evaluate access capacity based on marketing - distribution dispatching information fusion system. After extracting the relevant data from marketing distribution - dispatching information fusion system, calculating the maximum installed accessible capacity and the capacity accessed, we consider capacity bottleneck and power restoration between Transformers Area, branch lines and backbone lines to invent the method. In the last, based on this method the article calculate access capacity of a $10 \mathrm{kV}$ "hand in hand" distribution network line with double power source, the results show that this method can more accurately reflect how much are access capacity in distribution network line, it is effective and feasible.
\end{abstract}

\section{Introduction}

The access capacity [1] management refers to card the existed distribution network circuit, analyze various data through the control center, publish the access capacity, warnings and loads of distribution network regularly. With the advance of marketing-distribution-dispatching information fusion, the demand for service efficiency have become higher and higher. So the research on access capacity in the distribution network becomes more and more urgent.

For the distribution network, the current results mainly are on Maximum power supply capacity. The methods of calculation are mainly include three stages[2-6]: the first stage was evaluating power supply capacity of distribution network based on the distribution capacity , such as the method of capacity-load ratio; the second stage was evaluating power supply capacity of distribution network based on power restoration in distribution network, such as maximum load multiples method and maximum network flow method; the third stage was evaluating power supply capacity of distribution network based on $\mathrm{N}-1$ security criteria in distribution Network, substation capacity and power restoration. For the study on access capacity of the distribution network, especially the calculation of access capacity on Transformers Area, branch lines and backbone lines, there are few research findings.

The article mainly considers marketing-distribution-dispatching information fusion system, capacity bottleneck and the relationship between Transformers Area, branch lines and backbone lines to invent the evaluation method.

\section{Calculation of the maximum installed accessible capacity}

The maximum installed accessible capacity [7] of Transformers Area is rated capacity of distribution transformers. The multiplication of conductor ampacity [8] and voltage are the maximum installed accessible capacity of branch lines and backbone lines capacity. The voltage is $10 \mathrm{kV}$. The ampacity consider that a line is composed of some lines whose diameters are different. 


\section{Calculation of the capacity accessed}

(1) The forecasting accuracy of medium and Long-term power load forecasting of Transformers Area

GM $(1,1)$ model is widely used in the forecasting accuracy of medium and Long-term power load forecasting, it is a model consisting of first-order differential equations with a variable. From the forecast time, we take the date of annual electricity consumption for the past five consecutive years as the raw data to construct the last Grey forecasting model [9-12].

(2) Maximum access capacity of Transformers Area

Firstly, derived from the load factor formula and the maximum load formula, we deduce maximum access capacity [13-14] formula of Transformers Area. Then we calculate maximum continuous load rate and it is substituted into maximum access capacity formula.

1) The derivation of maximum access capacity

The load factor formula of distribution transformer:

$$
\beta=\frac{P_{\max }}{S_{N} \cos \theta}
$$

The maximum load formula of distribution transformer:

$$
P_{\max }=\sum P_{N i} K_{x}
$$

According to the two formulas above:

$$
\sum P_{N i}=\frac{\beta}{K_{x}} S_{N} \cos \theta
$$

In the type (3): $S_{N}$ is Rated Capacity of distribution transformer. $\cos \theta$ is Load power factor; $K_{x}$ is Demand coefficient

2) Calculate the maximum continuous load rate of Transformers Area

There are 96 sampling points of Load factor every day; we collect a load rate for 15 minutes. The calculation steps as follows:

(1)Count sampling points of the previous year.

(2) Number the 96 sampling points of the day and the last 7 sampling points of last day, starting from 1 to 103.then divide these date into 96 groups like $1-8,2-9,3-10, \cdots \cdots 96-103$. Calculate the average value of every group.

(3) Select the maximum number in the 96 groups to be the maximum average continuous load rate in the day and compare the daily maximum continuous load rate. We get the Maximum continuous load rate in a year.

(3) Calculate the capacity accessed of Transformers Area

The improved AHP [15] is a method includes expert knowledge, experience and information. It has been widely used in analysis and decision-making. We use the improved AHP to get the weight of long-term load forecasting and maximum access capacity as a and b. In the last, we use a, b, long-term load forecasting and maximum access capacity to get the capacity accessed.

(4) Calculate the capacity accessed of branch line and backbone line

According to topology model of marketing-distribution-dispatching information fusion system, including analysis of capacity bottleneck and power restoration between Transformers Area, branch lines and backbone lines is practical; the accessible capacity already of branch line and backbone line was got.

\section{Calculation Process of access capacity}

(1) Calculation of the predicted value of access capacity

Access capacity of Transformers Area equals the difference by rated capacity and the capacity accessed. That of branch lines and backbone lines equals the difference by the maximum installed 
accessible capacity and the capacity accessed.

(2) Calculation of access capacity

Based on topology model of marketing-distribution-dispatching information fusion system in distribution network, we consider capacity bottleneck and power restoration between Transformers Area, branch lines and backbone lines to calculate access capacity.

$P_{T}$ is the predicted value of access capacity of Transformers Area. $P_{F}$ is the predicted value of access capacity of branch lines. $P_{Z}$ is the predicted value of access capacity of backbone lines. $P_{t \min }, P_{f \min }, P_{z \min }$ are access capacity of Transformers Area, branch lines and backbone lines respectively. $P_{t \min }^{\prime}, P_{f \min }^{\prime}, P_{z \min }^{\prime}$ are access capacity that other distribution lines can provide Transformers Area, branch lines or backbone lines with respectively.

1) Access capacity of Transformers Area

(1) $P_{T}>P_{F}$, access capacity is $\min \left\{P_{F}, P_{t \min }+P_{t \min }^{\prime}\right\}$

(2) $P_{T} \leq P_{F}$, access capacity is $\min \left\{P_{T}, P_{t \min }+P_{t \min }^{\prime}\right\}$

2) Access capacity of branch lines

Access capacity is $\min \left\{P_{F}, P_{f \text { min }}+P_{f \min }^{\prime}\right\}$

3) Access capacity of backbone lines

Access capacity is $\min \left\{P_{Z}, P_{z \min }+P_{z \min }^{\prime}\right\}$

\section{Analysis of example}

We use a $10 \mathrm{kV}$ "hand in hand" distribution network line [16] as an example shown in Fig1.

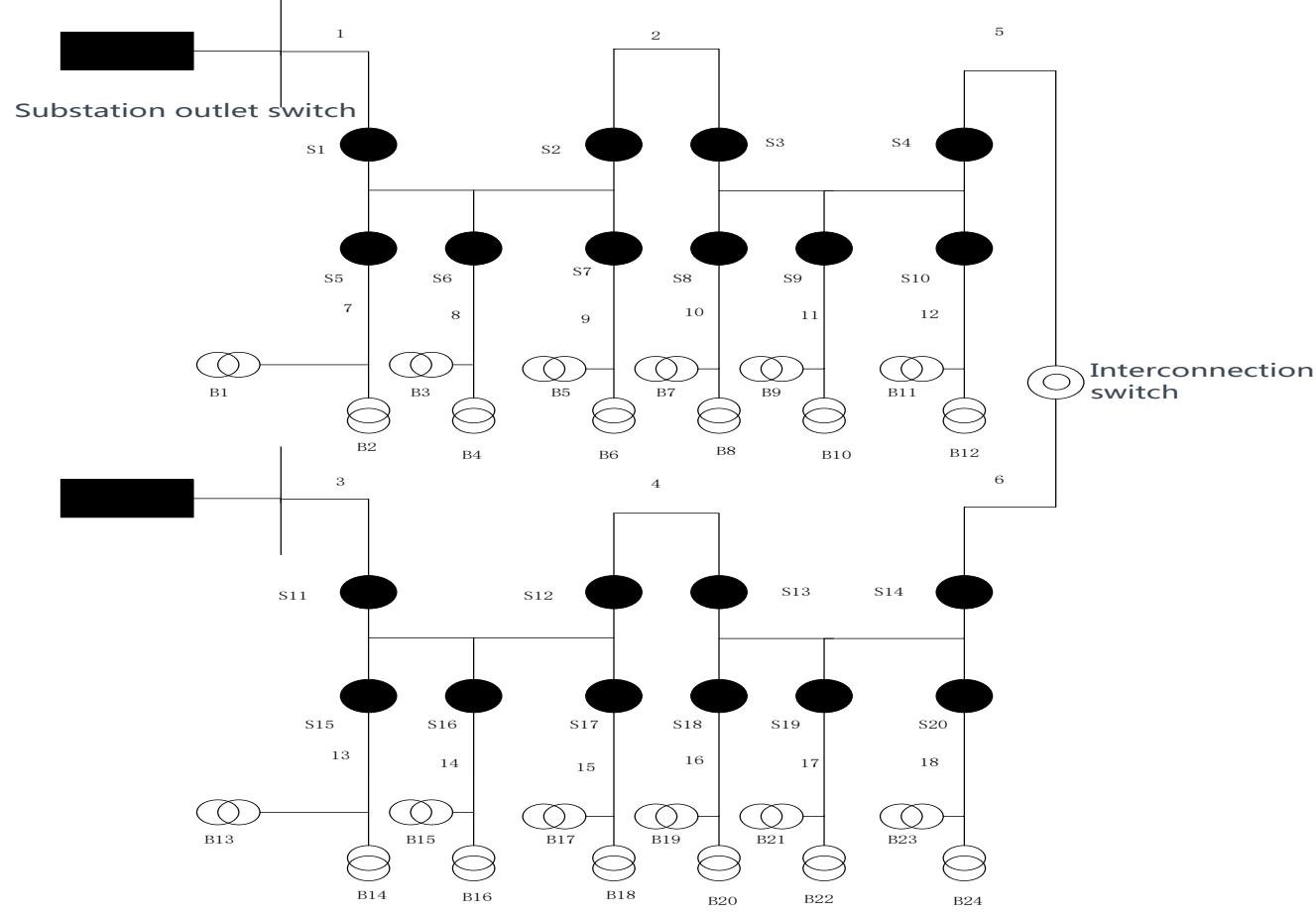

Fig.1. "hand in hand" distribution network line

In the figure above, B1-B24 represent Transformers Area, S1-S4 represent backbone lines and S7-S18 represent branch lines. It is assumed that the contact switch is turned off. The maximum installed accessible capacity of the $\mathrm{Bi}$ Transformers Area is $P_{b i}$, the capacity accessed of that is $P_{b i}^{\prime}$ and the expected value of access capacity is $P_{b i}^{\prime \prime}$. The maximum installed accessible capacity of the $\mathrm{i}$ line is $P_{s i}$, the capacity accessed of that is $P_{s i}{ }^{\prime}$, the expected value of access capacity is $P_{s i}{ }^{\prime \prime}$. The serial number of backbone lines is 1-4, The serial number of branch lines is 7-18。

The following is calculating the access capacity of Transformers Area branch lines and 
backbone lines.

(1) The access capacity of Transformers Area

B6 as an example:

$$
P_{t \min }=P_{s 1}^{\prime \prime}, P_{t \min }^{\prime}=\min \left\{P_{s 2}^{\prime \prime}, P_{s 3}^{\prime \prime}, P_{s 4}^{\prime \prime}\right\}
$$

1) $P_{b 6}{ }^{\prime \prime}>P_{s 9}{ }^{\prime \prime}$, the access capacity of B6 is $\min \left\{P_{s 9}{ }^{\prime \prime}, P_{t \min }+P_{t \min }{ }^{\prime}\right\}$

2) $P_{b 6}{ }^{\prime \prime} \leq P_{s 9}{ }^{\prime \prime}$, the access capacity of B6 is $\min \left\{P_{b 6}{ }^{\prime \prime}, P_{t \min }+P_{t \min }{ }^{\prime}\right\}$

(2) The access capacity of branch lines

Number 11 branch line as an example.

$P_{f \min }=\min \left\{P_{s 1}^{\prime \prime}, P_{s 2}{ }^{\prime \prime}\right\}, \quad P_{f \min }^{\prime}=\min \left\{P_{s 3}{ }^{\prime \prime}, P_{s 4}{ }^{\prime \prime}\right\}$

The access capacity of number 11 branch line is $\min \left\{P_{s 11}{ }^{\prime \prime}, P_{f \min }+P_{f \min }^{\prime}\right\}$

(3) The access capacity of backbone lines

Number 2 backbone line as an example

$P_{z \min }=P_{s 1}^{\prime \prime}, \quad P_{z \min }^{\prime}=\min \left\{P_{s 3}{ }^{\prime \prime}, P_{s 4}{ }^{\prime \prime}\right\}$

The access capacity of number 2 backbone line is $\min \left\{P_{s 2}{ }^{\prime \prime}, P_{z \min }+P_{z \min }^{\prime}\right\}$

\section{Conclusion}

(1) When calculating ampacity of branch lines and backbone lines, we consider the fact that a line is composed of some lines whose diameters are different. The sampling points of load factor are calculated in group, we can get the more accurate value of the maximum continuous load factor of Transformers Area.

(2) When calculating the capacity accessed, we put different weight on the long-term load forecasting and maximum access capacity based on the improved AHP, we can get the more accurate value.

(3) The evaluation method considering topology model, capacity bottleneck and power restoration between Transformers Area, branch lines and backbone lines is practical. In the last, the article calculate access capacity of a $10 \mathrm{kV}$ "hand in hand" distribution network line with double power source, the results show that the method is effective and feasible.

\section{Acknowledgements}

This work is supported by National Natural Science Foundation of China under Grant No.61173108 and 61472128, Hunan Provincial Natural Science Foundation of China No.10JJ2045 as well as $14 \mathrm{JJ} 2150$ and State Grid Corporation Science and technology projects of China No.5216A514001K.

\section{References}

[1] Zheng Tao, Jia Shilong, Pan Yumei, Yang Guosheng. Research on allowed penetration level of DGs based on the traditional fault location methods [J]. Power System Technology, 2014,38 (8) : 2257-2262

[2] Gu Wenzhuo. Definition, model and calculation method of total supply capability of distribution system [D]. Tianjin: Tianjin University, 2012.

[3] Xiao Jun, Gong Xiaoxu, Wang Chengshan. Comparative validation between the maximum 
supply capacity of the distribution and N-1 security Calibration [J]. Automation of Electric Power Systems, 2012,36(18): 86-91

[4] Xiao Jun, Guo Xiaodan, Wang Chengshan, Zu Guoqiang. The nature of the maximum distribution network power supply capacity model solution [J]. Automation of Electric Power Systems,2013,37(16): 59-65

[5] $\mathrm{Yu} \mathrm{Xu,} \mathrm{Zhi-Ming} \mathrm{Chen.} \mathrm{Evaluation} \mathrm{of} \mathrm{power} \mathrm{supply} \mathrm{capability} \mathrm{in} \mathrm{medium} \mathrm{voltage} \mathrm{distribution}$ networks based on fuzzy theory [C]. Guilin: Machine Learning and Cybernetics,2011:10-13

[6] Jun Han,Shaoyun Ge,Hong Liu,Yang Liu,Xin Zhang. Structural optimization of urban distribution network for improving power supply capability [C]. Shanghai:Electricity Distribution (CICED),2012:10-14

[7] Li Qiang, Zhang Yang, Li Minghui. Research on the Reception of Power System for Wind Power Integration [J]. Power Electronics,2010, 44(6): 20-22

[8] Ma Guodong. The Current capacity of Wire and cable [M]. Beijin: China Water \& Power Press.2003

[9] Wen Jie.Research on regional long term power load forecasting model and its application [D].Baoding: North China Electric Power University, 2014

[10] Zhang Wei. A short-term load forecasting method of power network based on the human body comfort index [J]. Power System Protection and Control, 2013,41(9): 75-79

[11] Jian-Chang Lu, Xingping Zhang, Wei Sun. A Real-time Adaptive Forecasting Algorithm for Electric Power Load [C]. Dalian:Transmission and Distribution Conference and Exhibition,Asia and Pacific, 2005: 1-5

[12] Lidong Duan, Dongxiao Niu, Zhihong Gu. Long and Medium Term Power Load Forecasting with Multi-Level Recursive Regression Analysis[C].Shanghai:Intelligent Information Technology,2008: 20-22

[13] Liu Shengli, Feng Yueliang, Pan Bailang, Wu Tao. A study on the maximum access capacity of distribution transformer [J]. Electric engineering, 2014,4(7): 7-9

[14] Liu Shengli, Chen Jianhua, Cao Yang, Chen Gangguo. A study on the maximum access capacity of distribution network based on the N-1 and load characteristic [J].Power System Protection and Control, 2014,42(12): 126-130

[15] Guo Zong, Wei Gang, Li Ming, Chu Jiayuan. A power supply capability evaluation method of distributed power distribution network [J]. Modern electric power,2015,32(4): 56-61

[16] He Zhengyou. Analysis and application of the distribution network [M]. Beijin : Science press.2014 